\title{
Increasing interest for the Journal of Electrical Bioimpedance
}

\author{
Ørjan G. Martinsen and Sverre Grimnes
}

1. Editors-in-chief, Journal of Electrical Bioimpedance

2. Department of Physics, University of Oslo, Oslo, Norway

3. Department of Biomedical and Clinical Engineering, Rikshospitalet, Oslo University Hospital, Oslo, Norway

We are now entering the third year for the Journal of Electrical Bioimpedance. If we look at the statistics, we have a steady increase in the number of visitors to our journal. The figure below shows the number of unique visitors each month since we started counting (using Google Analytics) in March 2010. Many visitors return, so the total number of monthly visitors is significantly higher.

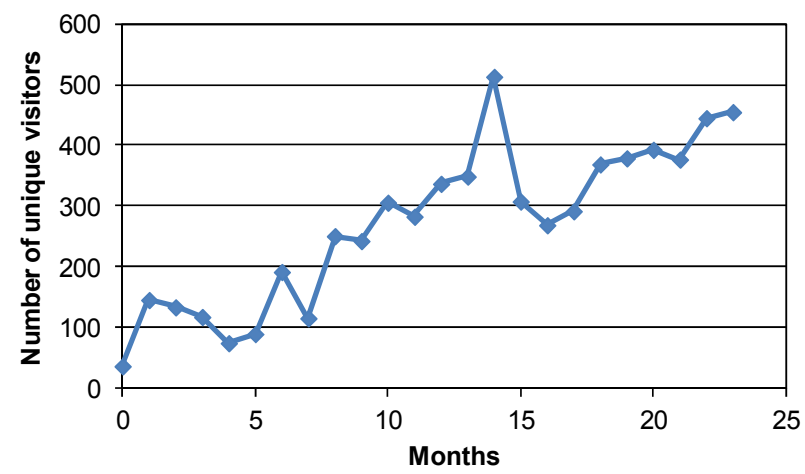

Fig.1: The number of unique monthly visitors at the JEB web site.

Figure 2 shows the geographical distribution of these visitors. As you can see, the interest for bioimpedance is quite global.

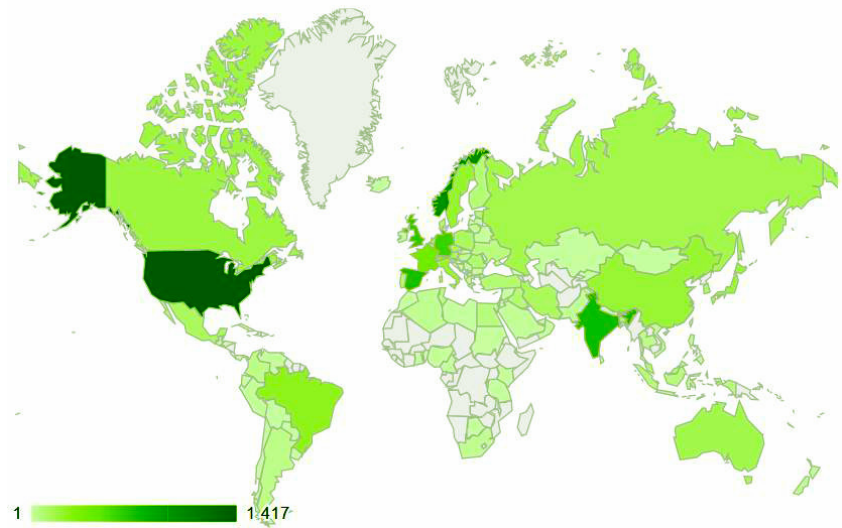

Fig.2: The geographical distribution of our readers.

Papers published in JEB have high visibility. All papers published in 2011 have every month been downloaded more than 100 times each.

There are also several activities to look forward to in the coming 12 months. The CLABIO 2012 - First Latin American Conference on Bioimpedance, is going to be held in Brazil, November 6-9 this year, and the triennial ICEBI will be held in conjunction with the XIV CEIT in Heilbad Heiligenstadt, Germany, April 22-25 in 2013. We hope to see you all at these conferences. 